\title{
Caputo type fractional difference operator and its application on discrete time scales
}

\author{
Mohamad Rafi Segi Rahmat ${ }^{*}$ and Mohd Salmi Md Noorani
}

"Correspondence:

Mohd.Rafi@nottingham.edu.my

'School of Applied Mathematics, The University of Nottingham

Malaysia Campus, Jalan Broga, Semenyih, Selangor Darul Ehsan 43500, Malaysia

Full list of author information is available at the end of the article

\begin{abstract}
In this paper, we introduce the analogue of Caputo type fractional derivatives on a $(q, h)$-discrete time scale which can be reduced to Caputo type fractional differences studied by Abdeljawad (Comput. Math. Appl. 62:1602-1611, 2011) and Caputo type fractional $q$-differences studied by Atici and Eloe via the choice $q=h=1$ and $h=0$, respectively. Then, we solve linear fractional difference equations involving Caputo type $(q, h)$-derivatives and give the general solutions in terms of discrete Mittag-Leffler functions introduced by Cermak et al. In addition, we also apply the $(q, h)$-Laplace transform method to solve these linear fractional order difference equations.
\end{abstract}

Keywords: fractional difference equations; discrete time scales; fractional calculus; $(q, h)$-calculus

\section{Introduction}

Fractional calculus deals with the study of fractional order integrals and derivatives and their applications [1-3]. Riemann-Liouville and Caputo are kinds of fractional derivatives which generalize the ordinary integral and differential operators. Differential equations with fractional derivative provided a natural framework for the discussion of various kinds of real problems modeled by the aid of fractional derivative, such as viscoelastic system, signal processing, diffusion processes, control processing, fractional stochastic system, allometry in biology and ecology (see [1, 4-7] and the references therein).

The study of fractional calculus in discrete settings has been initiated in [8-10]. While the papers $[8,10]$ present the introduction to fractional $q$-derivatives and $q$-integrals, the paper $[9,11]$ discusses the basics of fractional difference calculus. Among other significant papers dealing with these problems, we can mention, e.g., [4,12-15] or [16], where discrete analogues of some topics of continuous fractional calculus have been developed. For some recent contributions on fractional differential/difference equations, see [17-22] or [23] and the references therein.

The extension of basic notions of fractional calculus to other discrete settings was performed in $[24,25]$, where fractional sums and differences have been introduced and studied in the framework of $(q, h)$-calculus, which can be reduced to ordinary difference calculus and $q$-difference calculus via the choice $q=h=1$ and $h=0$, respectively. This extension follows recent trends in continuous and discrete analysis, characterized by a unification and generalization resulting into development of the time scales theory [26, 27]. Some interesting results concerning $(q, h)$-discrete time scales can be found in $[28,29]$.

(C) 2015 Segi Rahmat and Md Noorani. This article is distributed under the terms of the Creative Commons Attribution 4.0 International License (http://creativecommons.org/licenses/by/4.0/), which permits unrestricted use, distribution, and reproduction in any medium, provided you give appropriate credit to the original author(s) and the source, provide a link to the Creative Commons license, and indicate if changes were made. 
The aim of this paper is to introduce Caputo type nabla $(q, h)$-fractional difference operators and investigate their basic properties. Here, we solve some linear fractional difference equations involving Caputo type $(q, h)$-derivatives and provide the general solutions in terms of discrete Mittag-Leffler functions introduced in [25]. In addition, we also apply the $(q, h)$-Laplace transform method (introduced in [28]) to solve these linear fractional order difference equations.

\section{Preliminaries}

For the convenience of readers, we provide some basic concepts concerning the nabla calculus on time scales. The readers are referred to $[26,27,30]$ for further details of the time scale theory. By a time scale $\mathbb{T}$ we understand any nonempty, closed subset of reals with the ordering inherited from reals. Thus the reals $\mathbb{R}$, the integers $\mathbb{Z}$, the natural numbers $\mathbb{N}$, the nonnegative integers $\mathbb{N}_{0}$, the $h$-numbers $h \mathbb{Z}=\{h k: k \in \mathbb{Z}\}$ with fixed $h>0$, and the $q$-numbers $q^{\mathbb{N}_{0}}=\left\{q^{k}: k \in \mathbb{N}_{0}\right\}$ with fixed $q>1$ are examples of time scales.

For any $t \in \mathbb{T}$, we define the forward and backward jump operators as $\sigma(t):=\inf \{s \in$ $\mathbb{T}: s>t\}$ and $\rho(t):=\sup \{s \in \mathbb{T}: s<t\}$, respectively. The forward and backward graininess functions are defined as $\mu(t):=\sigma(t)-t$ and $\mu(t):=t-\rho(t)$, respectively. By convention, $\inf \{\emptyset\}=\sup \{\mathbb{T}\}$ and $\sup \{\emptyset\}=\inf \{\mathbb{T}\}$ for these operators. We say that a point is left-dense if $\rho(t)=t$ and left-scattered if $\rho(t) \neq t$. The right-dense and right-scattered points are defined in a similar manner. A point which is both left- and right-scattered is discrete. If $\inf \{\mathbb{T}\}=$ $a_{0}>-\infty$, we define $\mathbb{T}_{\kappa}:=\mathbb{T} \backslash a_{0}$, otherwise $\mathbb{T}_{\kappa}:=\mathbb{T}$.

For a function $f: \mathbb{T} \rightarrow \mathbb{R}$ and a point $t \in \mathbb{T}_{\kappa}$, we define $f^{\nabla}(t)$ to be a number such that for $\epsilon>0$ there exists a neighborhood $U \subset \mathbb{T}$ of $t$ which satisfies

$$
\left|f(\rho(t))-f(\tau)-f^{\nabla}(t)[\tau-\rho(t)]\right| \leq \epsilon|\tau-\rho(t)| \quad \text { for all } \tau \in U
$$

If $f^{\nabla}(t)$ is defined for all $t \in \mathbb{T}_{\kappa}$, then the function obtained is called the $\nabla$-derivative of $f$.

A function $f: \mathbb{T} \rightarrow \mathbb{R}$ is called left-dense continuous or ld-continuous provided it is continuous at every left-dense point in $\mathbb{T}$, and $f(t+)$ exists for every right-dense point in $\mathbb{T}$, and the set of ld-continuous functions is denoted by $C_{l d}(\mathbb{T})$. If $f \in C_{l d}(\mathbb{T})$, then there is a function $F$ such that $F^{\nabla}(t)=f(t)$. In this case, we define the $\nabla$-integral as

$$
\int_{a}^{t} f(\tau) \nabla \tau=F(t)-F(a) \quad \text { for all } t \in \mathbb{T} \text {. }
$$

Clearly, if $f: \mathbb{T} \rightarrow \mathbb{R}$ is ld-continuous and $t \in \mathbb{T}_{\kappa}$, then

$$
\int_{\rho(t)}^{t} f(\tau) \nabla \tau=v(t) f(t)
$$

A function $f \in C_{l d}(\mathbb{T})$ is called $v$-regressive if $1+f v \neq 0$ on $\mathbb{T}_{\kappa}$, and $f \in C_{l d}(\mathbb{T})$ is called positively regressive if $1+f v>0$ on $\mathbb{T}_{\kappa}$. The set of $v$-regressive functions and the set of positively $v$-regressive functions are denoted by $\mathcal{R}_{v}(\mathbb{T})$ and $\mathcal{R}_{v}^{+}(\mathbb{T})$, respectively. For simplicity, we denote by ${ }_{c} \mathcal{R}_{v}(\mathbb{T})$ the set of $\nu$-regressive constants. 
Let $\lambda \in{ }_{c} \mathcal{R}_{v}(\mathbb{T})$ and $s \in \mathbb{T}$, then the generalized exponential function $\widehat{e}_{\lambda}(\cdot, s)$ on time scale $\mathbb{T}$ is denoted by the unique solution of the initial value problem

$$
\left\{\begin{array}{l}
x^{\nabla}(t)=\lambda x(t), \quad t \in \mathbb{T}_{\kappa} ; \\
x(s)=1 .
\end{array}\right.
$$

For $p \in{ }_{c} \mathcal{R}_{v}(\mathbb{T})$, define circle minus $p$ by

$$
\ominus_{\nu} p:=-\frac{p}{1-p v}
$$

Then the unique solution of the initial value problem

$$
\left\{\begin{array}{l}
x^{\nabla}(t)=-\lambda x(t), \quad t \in \mathbb{T}_{k} \\
x(s)=1
\end{array}\right.
$$

takes the form $\widehat{\boldsymbol{e}}_{\ominus \nu}(\cdot, s)$. It is known that the exponential function $\widehat{\boldsymbol{e}}_{f}(\cdot, s)$ is strictly positive on $[s, \infty)_{\mathbb{T}}$ provided that $f \in \mathcal{R}^{+}\left([s, \infty)_{\mathbb{T}}\right)$.

The definition of the generalized monomials $\widehat{h}_{n}: \mathbb{T} \times \mathbb{T} \rightarrow \mathbb{R}\left(n \in \mathbb{N}_{0}\right)$ is given by

$$
\widehat{h}_{n}(t, s)= \begin{cases}1, & n=0 \\ \int_{s}^{t} \widehat{h}_{n-1}(\tau, s) \nabla \tau, & n \in \mathbb{N}\end{cases}
$$

for $s, t \in \mathbb{T}$ (see [27]). If we let $\widehat{h}_{n}^{\nabla}(t, s)$ denote for each fixed $s \in \mathbb{T}$ the derivatives of $\widehat{h}_{n}^{\nabla}(t, s)$ with respect to $t$, then

$$
\widehat{h}_{n}^{\nabla}(t, s)=\widehat{h}_{n-1}(t, s) \quad \text { for } n \in \mathbb{N}, t \in \mathbb{T}_{\kappa} \text {. }
$$

An important relation between the generalized exponential function and the monomials is given by

$$
\widehat{e}_{\lambda}(t, s)=\sum_{k=0}^{\infty} \lambda^{k} \widehat{h}_{k}(t, s) \quad \text { for } s, t \in \mathbb{T} \text { with } t \geq s,
$$

where $\lambda \in \mathcal{R}_{c}(\mathbb{T})$.

(First mean value theorem for integrals [27].) Let $f$ and $g$ be bounded and $\nabla$-integrable functions on $[a, b]_{\mathbb{T}}$, and let $g$ be nonnegative (or nonpositive) on $[a, b]_{\mathbb{T}}$. Set $m=\inf \{f(t)$ : $\left.t \in[a, b)_{\mathbb{T}}\right\}$ and $M=\sup \left\{f(t): t \in[a, b)_{\mathbb{T}}\right\}$. Then there exists a real number $\eta$ satisfying $m \leq \eta \leq M$ such that

$$
\int_{a}^{b} f(t) g(t) \nabla t=\eta \int_{a}^{b} g(t) \nabla t
$$

For example, the function $\widehat{h}_{n}(t, s) \geq 0$ for all $t \geq s$ and $n \in \mathbb{N}_{0}$. Let $f$ be an integrable function on $[t, s] \subset \mathbb{T}$, and let $m$ and $M$ be the infimum and the supremum, respectively, of $f$ on $[t, s]$. Then there exists a number $K$ between $m$ and $M$ such that

$$
\int_{s}^{t} f(\tau) \widehat{h}_{n}(\tau, s) \nabla \tau=K \int_{s}^{t} \widehat{h}_{n}(\tau, s) \nabla \tau=K \widehat{h}_{n+1}(t, s) .
$$


The nabla Laplace transform of a function $f: \mathbb{T} \rightarrow \mathbb{R}$ is defined by [21]

$$
\mathcal{L}\{f\}(z, s):=\int_{s}^{\infty} \widehat{e}_{\ominus_{v} z}(\rho(\tau), s) f(\tau) \nabla \tau \quad \text { for } z \in \mathcal{D}
$$

where $\mathcal{D}$ consists of all complex numbers $z \in \mathbb{C}$ for which the improper $\nabla$-integral exists.

The convolution of two functions $f, g: \mathbb{T} \rightarrow \mathbb{R}$ is defined by

$$
(f * g)(t):=\int_{a}^{t} \widehat{f}(t, \rho(s)) g(s) \nabla s, \quad t \in \mathbb{T}
$$

where $\widehat{f}$ is the shift of $f$ introduced in [31]. Then

$$
\mathcal{L}\{f * g\}(z)=\mathcal{L}\{f\}(z) \cdot \mathcal{L}\{g\}(z)
$$

The following result is the nabla version of the result obtained in [20]: (change of integration order) Let $f \in C_{l d}\left(\mathbb{T}^{2}\right)$, then

$$
\int_{s}^{t} \int_{s}^{\eta} f(\eta, \zeta) \nabla \zeta \nabla \eta=\int_{s}^{t} \int_{\rho(\zeta)}^{t} f(\eta, \zeta) \nabla \eta \nabla \zeta \quad \text { for } s, t \in \mathbb{T} .
$$

\section{$3(q, h)$-Fractional calculus}

Consider the following $(q, h)$-time scale (for details, see [24, 25]):

$$
\mathbb{T}_{(q, h)}^{t_{0}}=\left\{t_{0} q^{k}+[k]_{q} h: k \in \mathbb{Z}\right\} \cup\left\{\frac{h}{1-q}\right\}
$$

for $t_{0}>0, q \geq 1, h \geq 0$ and $q+h>1$. Note that if $q=1$, then the cluster point $h /(1-q)=-\infty$ is not involved in $\mathbb{T}$. The forward and backward jump operator is the linear function $\sigma(t)=$ $q t+h$ and $\rho(t)=q^{-1}(t-h)$, respectively. Similarly, the forward and backward graininess is given by $\mu(t)=(q-1) t+h$ and $v(t)=q^{-1} \mu(t)$, respectively. Observe that

$$
\sigma^{k}(t)=q^{k} t+[k]_{q} h \quad \text { and } \quad \rho^{k}(t)=q^{-k}\left(t-[k]_{q} h\right) .
$$

The following relation

$$
v\left(\rho^{k}(t)\right)=q^{-k} v(t)
$$

holds for $t \in \mathbb{T}$.

The nabla $(q, h)$-derivative of the function $f: \mathbb{T}_{(q, h)}^{t_{0}} \rightarrow \mathbb{R}$ is defined by

$$
\nabla_{(q, h)} f(t):=\frac{f(t)-f(\rho(t))}{v(t)}=\frac{f(t)-f(\tilde{q}(t-h))}{(1-\tilde{q}) t+\tilde{q} h},
$$


where $\tilde{q}=q^{-1}$. Let $t, a \in \mathbb{T}_{(q, h)}^{t_{0}}$ such that $h /(1-q) \leq a \leq t$ and $f: \mathbb{T} \rightarrow \mathbb{R}$. Then the nabla $(q, h)$-integral exists and can be calculated (provided $t>a$ ) via the formula

$$
\begin{aligned}
{ }_{a} \nabla_{(q, h)}^{-1} f(t) & :=\int_{a}^{t} f(\tau) \nabla \tau=\sum_{k=0}^{n-1} v\left(\rho^{k}(t)\right) f\left(\rho^{k}(t)\right) \\
& =\left(\left(1-q^{-1}\right) t+q^{-1} h\right) \sum_{k=0}^{n-1} q^{-k} f\left(q^{-k} t+[-k]_{q} h\right) .
\end{aligned}
$$

The Taylor monomials and the power functions on $\mathbb{T}_{(q, h)}^{t_{0}}$ have the forms

$$
\begin{aligned}
\widehat{h}_{n}(t, s) & =\frac{\prod_{j=0}^{n-1}\left(\sigma^{j}(t)-s\right)}{[n]_{q} !}=\frac{\prod_{j=0}^{n-1}\left(t, \rho^{j}(s)\right)}{[n]_{\tilde{q}} !}, \\
(t-s)_{(\tilde{q}, h)}^{(n)} & =\prod_{j=0}^{n-1}\left(t-\rho^{j}(s)\right),
\end{aligned}
$$

respectively, and the extension of the monomials $\widehat{h}_{n}(t, s)$ corresponding to $\mathbb{T}_{(q, h)}^{t_{0}}$ takes the form

$$
\widehat{h}_{\alpha}(t, s)=\frac{(t-s)_{(\tilde{q}, h)}^{(\alpha)}}{\Gamma_{\tilde{q}}(\alpha+1)}, \quad \alpha \in \mathbb{R} .
$$

Another (equivalent) expression of $\widehat{h}_{\alpha}(t, s)$ is provided by the following assertion.

Proposition 3.1 Let $\alpha \in \mathbb{R}, s, t \in \mathbb{T}_{(q, h)}^{t_{0}}$ and $n \in \mathbb{N}_{0}$ be such that $t=\sigma^{n}(s)$. Then

$$
\begin{aligned}
\widehat{h}_{\alpha}(t, s) & =(v(t))^{\alpha}\left[\begin{array}{c}
\alpha+n-1 \\
n-1
\end{array}\right]_{\tilde{q}} \\
& =(v(t))^{\alpha}\left[\begin{array}{c}
-\alpha-1 \\
n-1
\end{array}\right]_{\tilde{q}}(-1)^{n-1} \tilde{q}^{\alpha(n-1)+\left(\begin{array}{c}
n \\
2
\end{array}\right) .}
\end{aligned}
$$

Proposition 3.2 Let $\alpha \in \mathbb{R}, s, t \in \mathbb{T}_{(q, h)}^{t_{0}}$ and $k, n \in \mathbb{N}_{0}$ be such that $t=\sigma^{n}(s)$ and $k \leq n$. Then

$$
\begin{aligned}
\widehat{h}_{\alpha}\left(\rho^{k}(t), s\right) & =\tilde{q}^{\alpha k}(v(t))^{\alpha}\left[\begin{array}{c}
\alpha+n-k-1 \\
n-k-1
\end{array}\right]_{\tilde{q}} \\
& =\tilde{q}^{\alpha k}(v(t))^{\alpha}\left[\begin{array}{c}
-\beta \\
n-k-1
\end{array}\right]_{\tilde{q}}(-1)^{n-k-1} \tilde{q}^{(\beta-1)(n-k-1)+\left(\begin{array}{c}
n-k \\
2
\end{array}\right)} .
\end{aligned}
$$

Proof The proof is similar to the proof of Proposition 2.2 in [25].

Let $q>1$. Using the relations

$$
[t]=\frac{v(t)}{(1-\tilde{q})} \quad \Rightarrow \quad\left[\rho^{k}(t)\right]=\tilde{q}^{k} \frac{v(t)}{(1-\tilde{q})} \quad \text { and } \quad \frac{[s]}{\left[\rho^{k}(t)\right]}=\tilde{q}^{n-k}
$$


we can derive that

$$
\begin{aligned}
\widehat{h}_{\alpha}\left(\rho^{k}(t), s\right) & =\frac{\left[\rho^{k}(t)\right]^{\alpha}\left([s] /\left[\rho^{k}(t)\right], \tilde{q}\right)_{\infty}}{\Gamma_{\tilde{q}}(\alpha+1)\left(\tilde{q}^{\alpha}[s] /\left[\rho^{k}(t)\right], \tilde{q}\right)_{\infty}} \\
& =\frac{\tilde{q}^{\alpha k}(v(t))^{\alpha}(1-\tilde{q})^{-\alpha}\left(\tilde{q}^{n-k}, \tilde{q}\right)_{\infty}}{\Gamma_{\tilde{q}}(\alpha+1)\left(\tilde{q}^{\alpha+n-k}, \tilde{q}\right)_{\infty}} \\
& =\tilde{q}^{\alpha k}(v(t))^{\alpha} \frac{\Gamma_{\tilde{q}}(\alpha+n-k)}{\Gamma_{\tilde{q}}(\alpha+1) \Gamma_{\tilde{q}}(n-k)} \\
& =\tilde{q}^{\alpha k}(v(t))^{\alpha}\left[\begin{array}{c}
\alpha+n-k-1 \\
n-k-1
\end{array}\right]_{\tilde{q}} \\
& =\tilde{q}^{\alpha k}(v(t))^{\alpha}\left[\begin{array}{c}
-\beta \\
n-k-1
\end{array}\right]_{\tilde{q}}(-1)^{n-k-1} \tilde{q}^{(\beta-1)(n-k-1)+\left(\begin{array}{c}
(n-k) \\
2
\end{array}\right) .}
\end{aligned}
$$

Here, we use the identities

$$
\begin{aligned}
& {\left[\begin{array}{l}
x \\
k
\end{array}\right]_{\tilde{q}}=\frac{\Gamma_{\tilde{q}}(x+1)}{\Gamma_{\tilde{q}}(k+1) \Gamma_{\tilde{q}}(x-k+1)}, \quad x \in \mathbb{R}, k \in \mathbb{Z},} \\
& \frac{\Gamma_{\tilde{q}}(x+m)}{\Gamma_{\tilde{q}}(x)}=(-1)^{m} \tilde{q}^{x m+\left(\begin{array}{c}
m \\
2
\end{array}\right)} \frac{\Gamma_{\tilde{q}}(1-x)}{\Gamma_{\tilde{q}}(1-x-m)}, \quad x \in \mathbb{R}, m \in \mathbb{Z}^{+},
\end{aligned}
$$

where $\Gamma_{\tilde{q}}$ is the $q$-gamma function defined as

$$
\Gamma_{\tilde{q}}(x)=\frac{(\tilde{q}, \tilde{q})_{\infty}(1-\tilde{q})^{1-x}}{\left(\tilde{q}^{x}, \tilde{q}\right)_{\infty}}, \quad 0<\tilde{q}<1
$$

The following theorem is an extension of the result provided in [32] in $\mathbb{T}_{(q, h)}^{t_{0}}$.

Theorem 3.3 Let $n \in \mathbb{N}$ and $t, a \in \mathbb{T}_{(q, h)}^{t_{0}}$. Suppose $f: \mathbb{T}_{(q, h)}^{t_{0}} \rightarrow \mathbb{R}$, then

$$
f(t)=\sum_{k=0}^{n-1} \widehat{h}_{k}(t, a) \nabla_{(q, h)}^{k} f(a)+\int_{a}^{t} \widehat{h}_{n-1}(t, \rho(\tau)) \nabla_{(q, h)}^{n} f(\tau) \nabla \tau .
$$

Lemma 3.4 ([25]) Let $m \in \mathbb{Z}^{+}, \alpha \in \mathbb{R}, s, t \in \mathbb{T}_{(q, h)}^{t_{0}}$ and $n \in \mathbb{Z}^{+}, n \geq m$ be such that $t=\sigma^{n}(s)$. Then

$$
\nabla_{(q, h)}^{m} \widehat{h}_{\alpha}(t, s)= \begin{cases}\widehat{h}_{\alpha-m}(t, s), & \alpha \notin\{0,1, \ldots, m-1\}, \\ 0, & \alpha \in\{0,1, \ldots, m-1\} .\end{cases}
$$

Let $a \in \mathbb{T}_{(q, h)}^{t_{0}}, a>h /(1-q)$ be fixed. We consider the following restricted $(q, h)$-time scale:

$$
\widetilde{\mathbb{T}}_{(q, h)}^{\sigma^{i}(a)}=\left\{t \in \mathbb{T}, t \geq \sigma^{i}(a)\right\}, \quad i=0,1,2, \ldots
$$

where the symbol $\sigma^{i}$ stands for the $i$ th iterate of $\sigma$ (analogously, we use the symbol $\rho^{i}$ ).

Now we can continue with the introduction of $(q, h)$-fractional integral and $(q, h)$ fractional derivative of a function $f: \widetilde{\mathbb{T}}_{(q, h)}^{\sigma^{i}(a)} \rightarrow \mathbb{R}$. Let $t \in \widetilde{\mathbb{T}}_{(q, h)}^{\sigma^{i}(a)}$. 
Definition 3.5 ([25]) The nabla $(q, h)$-fractional integral (in the sense of RiemannLiouville) of order $\alpha \in \mathbb{R}^{+}$over the time scale interval $[a, t] \cap \widetilde{\mathbb{T}}_{(q, h)}^{a}$ is defined by

$$
{ }_{a} \nabla_{(q, h)}^{-\alpha} f(t):=\int_{a}^{t} \widehat{h}_{\alpha-1}(t, \rho(\tau)) f(\tau) \nabla \tau
$$

The nabla $(q, h)$-fractional derivative (in the sense of Caputo) of order $\alpha \in \mathbb{R}^{+}$is defined by

$$
\begin{aligned}
{ }_{a}^{C} \nabla_{(q, h)}^{\alpha} f(t) & :={ }_{a} \nabla^{-(m-\alpha)} \nabla^{m} f(t) \\
& =\int_{a}^{t} \widehat{h}_{m-\alpha-1}(t, \rho(\tau)) \nabla^{m} f(\tau) \nabla \tau,
\end{aligned}
$$

where $m \in \mathbb{Z}^{+}$is such that $m-1<\alpha \leq m$.

Lemma 3.6 ([25]) Let $\alpha \in \mathbb{R}^{+}, \beta \in \mathbb{R}$ and $t \in \widetilde{\mathbb{T}}_{(q, h)}^{\sigma(a)}$. Then

$$
{ }_{a} \nabla_{(q, h)}^{-\alpha} \widehat{h}_{\beta}(t, a)=\widehat{h}_{\alpha+\beta}(t, a) .
$$

The following lemma is useful.

Lemma 3.7 For all $\alpha, \beta \in \mathbb{R}$ and $t, s \in \widetilde{\mathbb{T}}_{(q, h)}^{\sigma(a)}$,

$$
\int_{a}^{t} \widehat{h}_{\alpha-1}(t, \rho(\tau)) \widehat{h}_{\beta-1}(\tau, a) \nabla \tau=\widehat{h}_{\alpha+\beta-1}(t, a)
$$

The proof is similar to the proof of Proposition 4.1 in [25]. For the sake of completeness, we give the following proof.

Proof Let $\widetilde{T}_{(q, h)}^{\sigma(a)}$ be such that $t=\sigma^{n}(a)$ for some $n \in \mathbb{Z}^{+}$. We have

$$
\begin{aligned}
\int_{a}^{t} \widehat{h}_{\alpha-1}(t, \rho(\tau)) \widehat{h}_{\beta-1}(\tau, a) \nabla \tau \\
=\sum_{k=0}^{n-1} \widehat{h}_{\alpha-1}\left(t, \rho^{k+1}(t)\right) \widehat{h}_{\beta-1}\left(\rho^{k}(t), a\right) v\left(\rho^{k}(t)\right) \\
=\sum_{k=0}^{n-1}(v(t))^{\alpha-1}\left[\begin{array}{c}
-\alpha \\
k
\end{array}\right]_{\tilde{q}}(-1)^{k} \tilde{q}^{(\alpha-1) k+\left(\begin{array}{c}
k+1 \\
2
\end{array}\right)} \\
\quad \times \tilde{q}^{(\beta-1) k}(v(t))^{\beta-1}\left[\begin{array}{c}
-\beta \\
n-k-1
\end{array}\right]_{\tilde{q}}(-1)^{n-k-1} \tilde{q}^{(\beta-1)(n-k-1)+\left(\begin{array}{c}
n-k \\
2
\end{array}\right) \tilde{q}^{k} v(t)} \\
=(v(t))^{\alpha+\beta-1} \sum_{k=0}^{n-1}\left[\begin{array}{c}
-\alpha \\
k
\end{array}\right]_{\tilde{q}}\left[\begin{array}{c}
-\beta \\
n-k-1
\end{array}\right]_{\tilde{q}}(-1)^{n-1} \tilde{q}^{k^{2}-k(n-1)+k \alpha+\left(\begin{array}{c}
n \\
2
\end{array}\right)+(\beta-1)(n-1)} \\
=(v(t))^{\alpha+\beta-1} \sum_{k=0}^{n-1}\left[\begin{array}{c}
-\alpha \\
n-k-1
\end{array}\right]_{\tilde{q}}\left[\begin{array}{c}
-\beta \\
k
\end{array}\right]_{\tilde{q}} \\
\quad \times(-1)^{n-1} \tilde{q}^{(n-k-1)^{2}-(n-k-1)(n-1)+(n-k-1) \alpha+\left(\begin{array}{c}
n \\
2
\end{array}\right)+(\beta-1)(n-1)}
\end{aligned}
$$




$$
\begin{aligned}
& =(v(t))^{\alpha+\beta-1} \sum_{k=0}^{n-1}\left[\begin{array}{c}
-\alpha \\
n-k-1
\end{array}\right]_{\tilde{q}}\left[\begin{array}{c}
-\beta \\
k
\end{array}\right]_{\tilde{q}}(-1)^{n-1} \tilde{q}^{k^{2}-k(n-1)-k \alpha+(\alpha+\beta)(n-1)+\left(\begin{array}{l}
n \\
2
\end{array}\right)} \\
& =(v(t))^{\alpha+\beta-1} \sum_{k=0}^{n-1}\left[\begin{array}{c}
-\alpha \\
(n-1)-k
\end{array}\right]_{\tilde{q}}\left[\begin{array}{c}
-\beta \\
k
\end{array}\right]_{\tilde{q}}(-1)^{n-1} \tilde{q}^{k^{2}-k(n-1)-k \alpha+(\alpha+\beta-1)(n-1)+\left(\begin{array}{l}
n \\
2
\end{array}\right)} \\
& =(v(t))^{\alpha+\beta-1} \sum_{k=0}^{n-1}\left[\begin{array}{c}
-(\alpha+\beta-1)-1 \\
n-1
\end{array}\right]_{\tilde{q}}(-1)^{n-1} \tilde{q}^{(\alpha+\beta-1)(n-1)+\left(\begin{array}{c}
n \\
2
\end{array}\right)} \\
& =\widehat{h}_{\alpha+\beta-1}(t, a),
\end{aligned}
$$

where we used the $q$-Vandermonde identity [25]

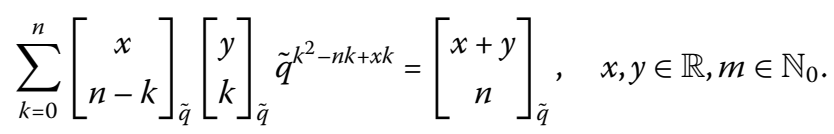

The following relations are used in the above proof:

$$
v\left(\rho^{k}(t)\right)=\tilde{q}^{k} v(t)
$$

With the power rule stated in Lemma 3.4, we can perform its extension to Caputo type nabla $(q, h)$-fractional derivative of the function $\widehat{h}_{\beta}(t, a)$ as below.

Lemma 3.8 Let $\alpha \in \mathbb{R}^{+}, \beta \in \mathbb{R}, t \in \widetilde{\mathbb{T}}_{(q, h)}^{\sigma^{m+1}(a)}$, where $m \in \mathbb{Z}^{+}$satisfies $m-1<\alpha \leq m$. Then

$$
{ }_{a}^{C} \nabla_{(q, h)}^{\alpha} \widehat{h}_{\beta}(t, a)= \begin{cases}\widehat{h}_{\beta-\alpha}(t, a), & \beta \notin\{0,1, \ldots, m-1\} ; \\ 0, & \beta \in\{0,1, \ldots, m-1\} .\end{cases}
$$

Proof The definition of Caputo nabla derivative and Lemma 3.4 yield

$$
\begin{aligned}
{ }_{a}^{C} \nabla_{(q, h)}^{\alpha} \widehat{h}_{\beta}(t, a) & ={ }_{a} \nabla^{-(m-\alpha)} \nabla^{m} \widehat{h}_{\beta}(t, a) \\
& ={ }_{a} \nabla^{-(m-\alpha)} \begin{cases}\widehat{h}_{\beta-m}(t, a), & \beta \notin\{0,1, \ldots, m-1\} ; \\
0, & \beta \in\{0,1, \ldots, m-1\},\end{cases} \\
& = \begin{cases}\widehat{h}_{\beta-\alpha}(t, a), & \beta \notin\{0,1, \ldots, m-1\} ; \\
0, & \beta \in\{0,1, \ldots, m-1\} .\end{cases}
\end{aligned}
$$

Theorem 3.9 Let $\alpha \in \mathbb{R}$ and $n \in \mathbb{Z}^{+}$so that $n-1<\alpha \leq n$. Then

$$
{ }_{a} \nabla_{(q, h) a}^{-\alpha} \nabla_{(q, h)}^{\alpha} f(t)=f(t)-\sum_{k=0}^{n-1} \widehat{h}_{k}(t, a) \nabla_{(q, h)}^{k} f(a) .
$$

Proof By deploying the definitions of fractional integral and Caputo nabla derivative, Lemma 3.7 and change of integration order, we obtain

$$
\begin{aligned}
{ }_{a} \nabla_{(q, h) a}^{-\alpha C} \nabla_{(q, h)}^{\alpha} f(t) & =\int_{a}^{t} \widehat{h}_{\alpha-1}(t, \rho(\tau))\left({ }_{a}^{C} \nabla_{(q, h)}^{\alpha} f(\tau)\right) \nabla \tau \\
& =\int_{a}^{t} \widehat{h}_{\alpha-1}(t, \rho(\tau))\left(\int_{a}^{\tau} \widehat{h}_{m-\alpha-1}(\tau, \rho(u))\left(\nabla_{(q, h)}^{m} f(u)\right) \nabla u\right) \nabla \tau
\end{aligned}
$$




$$
\begin{aligned}
& =\int_{a}^{t} \nabla_{(q, h)}^{m} f(u)\left(\int_{\rho(u)}^{t} \widehat{h}_{\alpha-1}(t, \rho(\tau)) \widehat{h}_{m-\alpha-1}(\tau, \rho(u)) \nabla \tau\right) \nabla u \\
& \left.=\int_{a}^{t} \widehat{h}_{m-1}(t, \rho(u)) \nabla_{(q, h)}^{m} f(u)\right) \nabla u \\
& =f(t)-\sum_{k=0}^{m-1} \widehat{h}_{k}(t, a) \nabla_{(q, h)}^{k} f(a) \quad \text { (by Taylor's formula, (16)). }
\end{aligned}
$$

In the following theorem we give the alternative definition of nabla $(q, h)$-derivative (Caputo).

Theorem 3.10 Let $\alpha \in \mathbb{R}$ and $n \in \mathbb{Z}^{+}$so that $n-1<\alpha \leq n$. Then

$$
{ }_{a}^{C} \nabla_{(q, h)}^{\alpha} f(t)={ }_{a} \nabla_{(q, h)}^{\alpha}\left\{f(t)-\sum_{k=0}^{n-1} \widehat{h}_{k}(t, a) \nabla_{(q, h)}^{k} f(a)\right\} .
$$

Proof It follows immediately from relation (14) and the fact that ${ }_{a} \nabla^{\alpha}\left({ }_{a} \nabla^{-\alpha} f\right)(t)=f(t)$ [24].

\section{Application}

In this section we give solutions for fractional initial value problems on $\mathbb{T}_{(q, h)}^{t_{0}}$. The following definition and results are due to Čermák et al. [25].

Definition 4.1 Let $\alpha, \beta, \lambda \in \mathbb{R}$. The $(q, h)$-Mittag-Leffler function $E_{\alpha, \beta}^{s, \lambda}(t)$ is defined by

$$
E_{\alpha, \beta}^{s, \lambda}(t)=\sum_{k=0}^{\infty} \lambda^{k} \widehat{h}_{\alpha k+\beta-1}(t, s) \quad\left(=\sum_{k=0}^{\infty} \lambda^{k} \frac{(t-s)_{(\tilde{q}, h)}^{(\alpha k+\beta-1)}}{\Gamma_{\tilde{q}}(\alpha k+\beta)}\right)
$$

for $s, t \in \widetilde{\mathbb{T}}_{(q, h)}^{\sigma(a)}$ and $t \geq s$.

It is easy to check that the series on the right-hand side converges (absolutely) if $|\lambda|(v(t))^{\alpha}<1$.

Theorem 4.2 ([25]) Let $\eta \in \mathbb{R}^{+}$and $t \in \mathbb{T}_{(q, h)^{\circ}}^{\sigma(a)}$. Then

$$
{ }_{a} \nabla_{(q, h)}^{-\eta} E_{\alpha, \beta}^{a, \lambda}(t)=E_{\alpha, \beta+\eta}^{a, \lambda}(t)
$$

Theorem 4.3 Let $\gamma \in \mathbb{R}^{+}, m \in \mathbb{Z}^{+}$be such that $m-1<\gamma \leq m$, and let $\alpha k+\beta-1 \notin$ $\{1,2, \ldots, m\}$ for all $k \in \mathbb{Z}^{+}$. If $t \in \mathbb{T}_{(q, h)}^{\sigma^{m+1}(a)}$, then

$$
{ }_{a}^{C} \nabla_{(q, h)}^{\gamma} E_{\alpha, \beta}^{a, \lambda}(t)= \begin{cases}E_{\alpha, \beta-\gamma}^{a, \lambda}(t), & \beta \notin\{1,2, \ldots, m\}, \\ \lambda E_{\alpha, \alpha+\beta-\gamma}^{a, \lambda}(t), & \beta \in\{1,2, \ldots, m\} .\end{cases}
$$

Proof By definition of $(q, h)$-Mittag-Leffler function,

$$
{ }_{a}^{C} \nabla_{(q, h)}^{\gamma} E_{\alpha, \beta}^{a, \lambda}(t)={ }_{a}^{C} \nabla_{(q, h)}^{\gamma} \sum_{k=0}^{\infty} \lambda^{k} \widehat{h}_{\alpha}(t, s)_{\alpha k+\beta-1}(t, a)=\sum_{k=0}^{\infty} \lambda_{a}^{k C} \nabla_{(q, h)}^{\gamma} \widehat{h}_{\alpha}(t, s)_{\alpha k+\beta-1}(t, a)
$$

due to the absolute convergence property. 
If $k \in \mathbb{Z}^{+}$, then Lemma 3.8 implies the relation

$$
{ }_{a}^{C} \nabla_{(q, h)}^{\gamma} \widehat{h}_{\alpha}(t, s)_{\alpha k+\beta-1}(t, a)=\widehat{h}_{\alpha}(t, s)_{\alpha k+\beta-\gamma-1}(t, a)
$$

due to the assumption $\alpha k+\beta-1 \notin\{1,2, \ldots, m\}$. If $k=0$, then two possibilities may occur. If $\beta \notin\{1,2, \ldots, m\}$, we get that (20) with $k=0$ implies the validity of $(18)_{1}$. If $\beta \in\{1,2, \ldots, m\}$, then ${ }_{a}^{C} \nabla_{(q, h)}^{\gamma} \widehat{h}_{\alpha}(t, s)_{\alpha k+\beta-1}(t, a)=0$, and by shifting the index $k$, i.e.,

$$
\sum_{k=1}^{\infty} \lambda_{a}^{k C} \nabla_{(q, h)}^{\gamma} \widehat{h}_{\alpha}(t, s)_{\alpha k+\beta-1}(t, a)=\lambda \sum_{k=0}^{\infty} \lambda^{k} \widehat{h}_{\alpha}(t, s)_{\alpha k+\alpha+\beta-\gamma-1}(t, a),
$$

we obtain $(18)_{2}$. This completes the proof.

Let us consider the following Cauchy type fractional initial value problem (FIVP):

$$
\begin{aligned}
& { }_{a}^{C} \nabla_{(q, h)}^{\alpha} y(t)-\lambda y(t)=f(t), \quad t \in \widetilde{\mathbb{T}}_{(q, h)}^{\sigma^{n+1}(a)}, \\
& \left.\nabla^{j} y(t)\right|_{t=\sigma^{n-1}(a)}=b_{j} \in \mathbb{R}, \quad j=0,1, \ldots, n-1,
\end{aligned}
$$

where $f: \widetilde{\mathbb{T}}_{(q, h)}^{\sigma^{n+1}(a)} \rightarrow \mathbb{R}, \alpha \in \mathbb{R}^{+}$and $n \in \mathbb{Z}^{+}$are such that $n-1<\alpha \leq n$. Here, we assume that $v$-regressivity condition is ensured, that is, $\lambda(v(t))^{\alpha} \neq 1$.

It can be easily verified that the above FIVP can be reduced to the Volterra type integral equation

$$
y(t)=\sum_{k=0}^{n-1} b_{k} \widehat{h}_{k}(t, a)+\lambda \int_{a}^{t} \widehat{h}_{\alpha-1}(t, \rho(\tau)) y(\tau) \nabla \tau+\int_{a}^{t} \widehat{h}_{\alpha-1}(t, \rho(\tau)) f(\tau) \nabla \tau .
$$

We apply the method of successive approximations by setting

$$
\begin{aligned}
& y_{0}(t)=\sum_{k=0}^{n-1} b_{k} \widehat{h}_{k}(t, a), \\
& y_{m}(t)=y_{0}(t)+\lambda \int_{a}^{t} \widehat{h}_{\alpha-1}(t, \rho(\tau)) y_{m-1}(\tau) \nabla \tau+\int_{a}^{t} \widehat{h}_{\alpha-1}(t, \rho(\tau)) f(\tau) \nabla \tau .
\end{aligned}
$$

Thus

$$
\begin{aligned}
y_{1}(t) & =\sum_{k=0}^{n-1} b_{k} \widehat{h}_{k}(t, a)+\sum_{k=0}^{n-1} \lambda b_{k} \widehat{h}_{\alpha+k}(t, a)+\int_{a}^{t} \widehat{h}_{\alpha-1}(t, \rho(\tau)) f(\tau) \nabla \tau \\
& =\sum_{k=0}^{n-1} b_{k} \sum_{j=0}^{1} \lambda \widehat{h}_{\alpha j+k}(t, a)+\int_{a}^{t} \widehat{h}_{\alpha-1}(t, \rho(\tau)) f(\tau) \nabla \tau, \\
y_{2}(t) & =\sum_{k=0}^{n-1} b_{k} \sum_{j=0}^{2} \lambda^{\widehat{h}} \widehat{h}_{\alpha j+k}(t, a)+\int_{a}^{t}\left[\lambda \widehat{h}_{2 \alpha-1}(t, \rho(\tau))+\widehat{h}_{\alpha-1}(t, \rho(\tau))\right] f(\tau) \nabla \tau \\
& =\sum_{k=0}^{n-1} b_{k} \sum_{j=0}^{2} \lambda \widehat{h}_{\alpha j+k}(t, a)+\int_{a}^{t} \sum_{j=1}^{2} \lambda^{j-1} \widehat{h}_{\alpha j-1}(t, \rho(\tau)) f(\tau) \nabla \tau,
\end{aligned}
$$




$$
y_{m}(t)=\sum_{k=0}^{n-1} b_{k} \sum_{j=0}^{m} \lambda^{j} \widehat{h}_{\alpha j+k}(t, a)+\int_{a}^{t} \sum_{j=1}^{m} \lambda^{j-1} \widehat{h}_{\alpha j-1}(t, \rho(\tau)) f(\tau) \nabla \tau .
$$

Taking the limit as $m \rightarrow \infty$, we arrive at the approximate solution to equation (24), and taking into account (16), we obtain

$$
y(t)=\sum_{k=0}^{n-1} b_{k} E_{\alpha, k+1}^{a, \lambda}(t)+\int_{a}^{t} E_{\alpha, \alpha}^{\rho(\tau), \lambda}(\tau) f(\tau) \nabla \tau .
$$

This yields an explicit solution $y(t)$ of the Volterra integral equation (24) and hence a solution for (22)-(23).

Clearly, the Cauchy problem (22) involving the homogeneous equation

$$
{ }_{a}^{C} \nabla_{(q, h)}^{\alpha} y(t)-\lambda y(t)=0, \quad t \in \widetilde{\mathbb{T}}_{(q, h)}^{\sigma^{n+1}(a)}, n-1<\alpha \leq n
$$

has a unique solution of the form

$$
y(t)=\sum_{k=0}^{n-1} b_{k} E_{\alpha, k+1}^{a, \lambda}(t)
$$

Corollary 4.4 The solution of the Cauchy problem

$$
\begin{aligned}
& { }_{a}^{C} \nabla_{(q, h)}^{\alpha} y(t)-\lambda y(t)=f(t), \quad t \in \widetilde{\mathbb{T}}_{(q, h)}^{\sigma^{2}(a)}, 0<\alpha<1, \\
& y(a)=1 \in \mathbb{R}
\end{aligned}
$$

has the form

$$
y(t)=E_{\alpha, 1}^{a, \lambda}(t)+\int_{a}^{t} E_{\alpha, \alpha}^{\rho(\tau), \lambda}(\tau) f(\tau) \nabla \tau
$$

while the solution to the problem

$$
\begin{aligned}
& { }_{a}^{C} \nabla_{(q, h)}^{\alpha} y(t)=\lambda y(t), \quad t \in \widetilde{\mathbb{T}}_{(q, h)}^{\sigma^{2}(a)}, 0<\alpha<1, \\
& y(a)=1 \in \mathbb{R}
\end{aligned}
$$

is given by

$$
y(t)=E_{\alpha, 1}^{a, \lambda}(t)
$$

Finally, we give the Laplace transforms of fractional nabla integral and Caputo nabla derivative on $\mathbb{T}_{(q, h)}^{t_{0}}$. For $t, s \in \mathbb{T}_{(q, h)}^{t_{0}}$ and $\alpha \in \mathbb{R}^{+}$, we have [21]

$$
\widehat{\mathcal{L}}_{(q, h)}\left\{\widehat{h}_{\alpha}(t, s)\right\}(z)=\frac{1}{z^{\alpha+1}} .
$$


From the definition of $(q, h)$-Mittag-Leffler function $E_{\alpha, \beta}^{s, \lambda}(t)$, we have

$$
\begin{aligned}
\widehat{\mathcal{L}}_{(q, h)}\left\{E_{\alpha, \beta}^{a, \lambda}\right\}(z) & =\widehat{\mathcal{L}}_{(q, h)}\left\{\sum_{k=0}^{\infty} \lambda^{k} \widehat{h}_{\alpha k+\beta-1}(\cdot, a)\right\}(z) \\
& =\sum_{k=0}^{\infty} \lambda^{k} \widehat{\mathcal{L}}_{(q, h)}\left\{\widehat{h}_{\alpha k+\beta-1}(\cdot, a)\right\}(z) \\
& =\sum_{k=0}^{\infty} \frac{\lambda^{k}}{z^{\alpha k+\beta}} \\
& =\frac{z^{\alpha-\beta}}{z^{\alpha}-\lambda} \quad \text { provided }\left|z^{-\alpha} \lambda\right|<1 .
\end{aligned}
$$

For $\alpha=1, \beta=1$ and $t \in \widetilde{\mathbb{T}}_{(q, h)}^{\sigma^{i}(a)}$, we have

$$
E_{1,1}^{a, \lambda}(t)=\widehat{e}_{\lambda}(t, a)
$$

Hence,

$$
\widehat{\mathcal{L}}_{(q, h)}\left\{\widehat{e}_{\lambda}(t, a)\right\}(z)=\widehat{\mathcal{L}}_{(q, h)}\left\{E_{1,1}^{a, \lambda}(t)\right\}=\frac{1}{z-\lambda} .
$$

Theorem 4.5 The $(q, h)$-Laplace transform of fractional nabla integral is given by

$$
\widehat{\mathcal{L}}_{(q, h)}\left\{{ }_{a} \nabla_{(q, h)}^{-\alpha} f\right\}(z)=z^{-\alpha} \widehat{\mathcal{L}}_{(q, h)}\{f\}(z) .
$$

Proof By convolution, one may write

$$
{ }_{a} \nabla_{(q, h)}^{-\alpha} f(t)=\int_{a}^{t} \widehat{h}_{\alpha-1}(t, \rho(\tau)) f(\tau) \nabla \tau=\left(\widehat{h}_{\alpha-1}(\cdot, a) * f\right)(t) .
$$

Thus,

$$
\begin{aligned}
\widehat{\mathcal{L}}_{(q, h)}\left\{{ }_{a} \nabla_{(q, h)}^{-\alpha} f\right\}(z) & =\widehat{\mathcal{L}}_{(q, h)}\left\{\widehat{h}_{\alpha-1}(t, a)\right\}(z) \cdot \widehat{\mathcal{L}}_{(q, h)}\{f\}(z) \\
& =\frac{1}{z^{\alpha}} \widehat{\mathcal{L}}_{(q, h)}\{f\}(z) .
\end{aligned}
$$

Theorem 4.6 The $(q, h)$-Laplace transform of Caputo nabla derivative is given by

$$
\widehat{\mathcal{L}}_{(q, h)}\left\{{ }_{a}^{C} \nabla_{(q, h)}^{\alpha} f\right\}(z)=z^{\alpha} \widehat{\mathcal{L}}_{(q, h)}\{f\}(z)-\sum_{k=0}^{m-1} z^{\alpha-k-1} \nabla_{(q, h)}^{k} f(a)
$$

where $m-1<\alpha \leq m$.

Proof Note that, in general (see Theorem 2.10 in [28]) for $n \in \mathbb{Z}^{+}$, we have

$$
\widehat{\mathcal{L}}_{(q, h)}\left\{\nabla_{(q, h)}^{n} f\right\}(z)=z^{n} \widehat{\mathcal{L}}_{(q, h)}\{f\}(z)-\sum_{i=0}^{n-1} z^{n-1-i} \nabla_{(q, h)}^{i} f(a) .
$$


Thus, for $m-1<\alpha \leq m$, in view of the definition of fractional derivative, we have

$$
\begin{aligned}
\widehat{\mathcal{L}}_{(q, h)}\left\{{ }_{a}^{C} \nabla_{(q, h)}^{\alpha} f\right\}(z) & =\widehat{\mathcal{L}}_{(q, h)}\left\{\widehat{h}_{m-\alpha-1}(\cdot, a)\right\}(z) \cdot \widehat{\mathcal{L}}_{(q, h)}\left\{\nabla_{(q, h)}^{m} f\right\}(z) \\
& =\frac{1}{z^{m-\alpha}}\left(z^{m} \widehat{\mathcal{L}}_{(q, h)}\{f\}(z)-\sum_{k=0}^{m-1} z^{m-1-k} \nabla_{(q, h)}^{k} f(a)\right) \\
& =z^{\alpha} \widehat{\mathcal{L}}_{(q, h)}\{f\}(z)-\sum_{k=0}^{m-1} z^{\alpha-k-1} \nabla_{(q, h)}^{k} f(a) .
\end{aligned}
$$

In particular, if $0<\alpha \leq 1$, then

$$
\widehat{\mathcal{L}}_{(q, h)}\left\{{ }_{a}^{C} \nabla_{(q, h)}^{\alpha} f\right\}(z)=z^{\alpha} \widehat{\mathcal{L}}_{(q, h)}\{f\}(z)-z^{\alpha-1} f(a) .
$$

As an application, we apply the Laplace transform method to derive explicit solutions to the homogeneous equations of the form

$$
{ }_{a}^{C} \nabla_{(q, h)}^{\alpha} y(t)-\lambda y(t)=0, \quad d_{k}=\nabla^{k} y(a), \quad k=0, \ldots, m-1,
$$

where $t \in \widetilde{\mathbb{T}}_{(q, h)}^{\sigma^{m}(a)}(m \in \mathbb{N}), m-1<\alpha \leq m, \lambda \in \mathbb{R}$, in terms of the $(q, h)$-Mittag-Leffler functions.

The following statement holds.

Theorem 4.7 Let $m \in \mathbb{N}$ be given by $m-1<\alpha \leq m$ and $\lambda \in \mathbb{R}$. Then the functions

$$
y_{i}(t)=E_{\alpha, i+1}^{a, \lambda}(t), \quad i=0,1, \ldots, m-1,
$$

yield the fundamental system of solutions to equation (42).

Proof Applying the Laplace transform to (42) and taking (39) into account, we have

$$
\widehat{\mathcal{L}}_{(q, h)}\{y\}(z)=\sum_{i=0}^{m-1} d_{k} \frac{z^{\alpha-i-1}}{z^{\alpha}-\lambda} .
$$

Formula (34) with $\beta=i+1$ yields

$$
\widehat{\mathcal{L}}_{(q, h)}\left\{E_{\alpha, i+1}^{a, \lambda}(t)\right\}(z)=\frac{z^{\alpha-i-1}}{z^{\alpha}-\lambda} .
$$

Thus, relation (44) yields

$$
y(t)=\sum_{i=0}^{m-1} d_{i} y_{i}(t), \quad \text { where } y_{i}(t)=E_{\alpha, i+1}^{a, \lambda}(t)
$$

\section{Corollary 4.8 The equation}

$$
{ }_{a}^{C} \nabla_{(q, h)}^{\alpha} y(t)-\lambda y(t)=0, \quad y(a)=1, \quad 0<\alpha \leq 1,
$$


has solution in the form

$$
y(t)=E_{\alpha, 1}^{a, \lambda}(t)
$$

while the equation

$$
{ }_{a}^{C} \nabla_{(q, h)}^{\alpha} y(t)-\lambda y(t)=0, \quad \nabla^{k} y(a)=1, \quad k=0,1,1<\alpha \leq 2,
$$

has its fundamental system of solutions given by

$$
y_{1}(t)=E_{\alpha, 1}^{a, \lambda}(t), \quad y_{2}(t)=E_{\alpha, 2}^{a, \lambda}(t) .
$$

\section{Competing interests}

The authors declare that they have no competing interests.

\section{Authors' contributions}

The main idea of this paper was proposed by SR and MS. SR prepared the manuscript initially and performed all the steps of the proofs in this research. All authors read and approved the final manuscript.

\section{Author details}

'School of Applied Mathematics, The University of Nottingham Malaysia Campus, Jalan Broga, Semenyih, Selangor Darul Ehsan 43500, Malaysia. ${ }^{2}$ School of Mathematical Science, Faculty of Science and Technology, University Kebangsaan Malaysia, Bangi, Selangor 43600, Malaysia.

\section{Acknowledgements}

The authors would like to thank Professor I Čermák and the anonymous referees for their valuable comments and suggestions. The authors would like to acknowledge the grants: UKM Grant DIP-2014-034 and Ministry of Education, Malaysia, Grant FRGS/1/2014/ST06/UKM/01/1 for financial support.

\section{Received: 2 December 2014 Accepted: 6 May 2015 Published online: 20 May 2015}

\section{References}

1. Kilbas, A, Srivastava, MH, Trujillo, Jj: Theory and Application of Fractional Differential Equations. Elsevier, Amsterdam (2006)

2. Miller, KS, Ross, B: An Introduction to the Fractional Calculus and Fractional Differential Equations. Wiley, New York (1993)

3. Podlubny, I: Fractional Differential Equations. Academic Press, San Diego (1999)

4. Guy, J: Modeling fractional stochastic systems an non-random fractional dynamics driven by Brownian motions. Appl. Math. Model. 32(5), 836-859 (2008)

5. Hilfer, R: Applications of Fractional Calculus in Physics. World Scientific, River Edge (2000)

6. Lakshmikantham, V, Leela, S, Devi, JV: Theory of Fractional Dynamical Systems. Cambridge Scientific Publishers, Cambridge (2009)

7. Li, C, Qian, D, Chen, YQ: On Riemann-Liouville and Caputo derivatives. Discrete Dyn. Nat. Soc. 2011, Article ID 562494 (2011)

8. Agarwal, RP: Certain fractional q-integrals and q-derivatives. Proc. Camb. Philos. Soc. 66, 365-370 (1969)

9. Díaz, JB, Osler, TJ: Differences of fractional order. Math. Comput. 28, 185-202 (1974)

10. Al-Salam, WA: Some fractional $q$-integrals and q-derivatives. Proc. Camb. Philos. Soc. 15, 135-140 (1966)

11. Grey, HL, Zhang, NF: On a new definition of the fractional difference. Math. Comput. 50, 513-529 (1988)

12. Atici, FM, Eloe, PW: A transform method in discrete fractional calculus. Int. J. Differ. Equ. 2, 165-176 (2007)

13. Atici, FM, Eloe, PW: Fractional q-calculus on a time scale. J. Nonlinear Math. Phys. 14(3), 341-352 (2007)

14. Atici, FM, Eloe, PW: Discrete fractional calculus with the nabla operator. Electron. J. Qual. Theory Differ. Equ. 2009, 3 (2009)

15. Fang, JP: Some applications of q-differential operator. J. Korean Math. Soc. 47(2), 223-233 (2010)

16. Mansour, ZSI: Linear sequential $q$-difference equations of fractional order. Fract. Calc. Appl. Anal. 12(2), 159-178 (2009)

17. Dassios, IK, Baleanu, DI: On a singular system of fractional nabla difference equations with boundary conditions. Bound. Value Probl. 2013, Article ID 148 (2013)

18. Jagan Mohan, J, Deekshitulu, GVSR: Solutions of nabla fractional difference equations using $N$-transforms. Commun. Math. Stat. 2, 1-16 (2014)

19. Jarad, F, Kaymakçalan, B, Teș, K: A new transform method in nabla discrete fractional calculus. Adv. Differ. Equ. 2012, Article ID 190 (2012)

20. Karpuz, B: Volterra theory on time scales. Results Math. 65, 263-292 (2014)

21. Segi Rahmat, MR: Integral transform method for solving fractional dynamic equations on time scales. Abstr. Appl. Anal. 2014, Article ID 261348 (2014)

22. Wu, G-C, Baleanu, D: New applications of the variational iteration method-from differential equations to $q$-fractional difference equations. Adv. Differ. Equ. 2013, Article ID 21 (2013) 
23. Abdeljawad, T: On Riemann and Caputo fractional differences. Comput. Math. Appl. 62, 1602-1611 (2011)

24. Čermák, J, Nechvátal, L: On (q,h)-analogue of fractional calculus. J. Nonlinear Math. Phys. 17(1), 1-18 (2010)

25. Čermák, J, Kisela, T, Nechvátal, L: Discrete Mittag-Leffler functions in linear fractional difference equations. Abstr. Appl. Anal. 2011, Article ID 565067 (2011)

26. Bohner, M, Peterson, A: Dynamic Equations on Time Scales: An Introduction with Applications. Birkhäuser, Boston (2001)

27. Bohner, M, Peterson, A (eds.): Advances in Dynamic Equations on Time Scales. Birkhäuser, Boston (2003)

28. Segi Rahmat, MR: The (q, h)-Laplace transform on discrete time scales. Comput. Math. Appl. 62, 272-281 (2011)

29. Segi Rahmat, MR: On some $(q, h)$-analogues of integral inequalities on discrete time scales. Comput. Math. Appl. 62, 1790-1797 (2011)

30. Agarwal, RP, Bohner, M: Basic calculus on time scales and some of its applications. Results Math. 35(1-2), 3-22 (1999)

31. Kisela, T: Power functions and essentials of fractional calculus on isolated time scales. Adv. Differ. Equ. 2013, Article ID 259 (2013)

32. Williams, PA: Fractional calculus on time scales with Taylor's theorem. Fract. Calc. Appl. Anal. 15(4), 616-638 (2012)

\section{Submit your manuscript to a SpringerOpen ${ }^{\circ}$ journal and benefit from:}

- Convenient online submission

Rigorous peer review

- Immediate publication on acceptance

- Open access: articles freely available online

- High visibility within the field

- Retaining the copyright to your article

Submit your next manuscript at springeropen.com 